\title{
Urethral Leiomyoma: A Case Report
}

\author{
(D) Murat Alan MD, (1) Mehmet Gökçü MD, (D Saime Ünlüoğlu MD, (1) Muzaffer Sancı MD \\ University of Health Sciences, Izmir Tepecik Training and Research Hospital, Clinic of Obstetrics and Gynecology, Izmir, Turkey
}

\begin{abstract}
Leiomyomas are benign smooth muscle uterine masses most frequently seen in females during the period when they can give birth. Extrauterine leiomyomas are seen rare and where they are observed the most is the genitourinary tract. In general, patients come up with urinary and soft tissue mass near the urethral meatus related problems. Here we report a case of distal urethral leiomyoma in a 40-year old woman who was admitted with urethral obstructive symptoms and was operated for periurethral mass. Leiomyomas originating from the smooth muscle of the urethra, a rare location, are generally diagnosed following surgery. These leiomyomas should be treated with great care during surgery due to their unusual location. Patients who undergo surgery due to the presence of urethral stricture can also be seen after myomectomy.
\end{abstract}

Keywords: Leiomyoma, urethra, distal urethral leiomyoma, muscle uterine mass

\section{Introduction}

Bladder and urethral leiomyomas are rare entities (1). Buttner (2) was the first person to report the first incidence of urethral leiomyoma. They may show various clinical symptoms depending on their site and size, which, at the urethral level forming especially in the adjacency of vaginal wall, include recurrent urinary tract infections, voiding dysfunction, foreign body sensation, pain and dyspareunia (3). Here we report a case of urethral leiomyoma in a patient with recurrent urological complaints. The leiomyoma was successfully removed by surgical resection without damaging the urinary tract and no recurrence has been detected to date.

\section{Case Report}

A 40-year-old woman with a history of urological complaints presented in 2017 with a perineal mass that had increased in size for 2 years, dyspareunia, and persistent dysuria. Urinalysis was negative for leukocytes and no growth was detected in urine culture. On physical examination, we detected a tender polypoid round perineal mass approximately $4 \mathrm{~cm}$ in diameter below the labia minora on the right side. Distant from the vaginal opening, the $4 \times 4 \mathrm{~cm}$ perineal mass extended in the anteroinferior direction from the urethral meatus. As the mass extended towards both the urethra and front of the vagina, it appeared to be excisable. Ultrasonography (USG) revealed that the uterus, bilateral ovaries, and bladder were normal.
The patient was informed about her condition and the benefits and potential risks of surgery. After obtaining written informed consent for the procedure, the patient underwent myomectomy under general anesthesia in sterile conditions.

Following the elevation of perineal mucosal flaps, local excision of the mass was performed with vaginal approach under spinal anesthesia. The mass was easily separated from the structures surrounding the anterior urethral wall using electrocautery. Vicryl 3/0 sutures were used to close up the mucosal tissues. Foley catheter was removed after 30 days and postoperative recovery was uneventful. The symptoms reported by the patient had fully resolved as of final follow-up at postoperative 1 year, no recurrence was observed, and the patient was asymptomatic. The mass had a macroscopic diameter of $2.5 \mathrm{~cm}$, smooth surface, and was pink and hard (Figure 1). In the examination of the mass, it was non-encapsulated, firm, gray-white, and showed no hemorrhage, necrosis, or cystic degeneration. Hematoxylin-eosin staining under microscopy revealed cells that appeared to be smooth muscle fascicules, which may be indicative of leiomyomas (Figure 2). The myoma tumor cells were regular and no atypia or mitosis were observed. Immunohistochemistry showed positive staining for vimentin, actin, and desmin in the tumor cells (Figure 3 ). Section image of the sample of well-encapsulated, solid, homogeneous mass appearing with no bleeding, necrosis, or cystic changes showed a leiomyoma tumor with muscle fiber areas.

Address for Correspondence: Murat Alan MD, University of Health Sciences, İzmir Tepecik Training and Research Hospital, Clinic of Obstetrics and Gynecology, İmir, Turkey E-mail: murat.alangozde@gmail.com ORCID-ID: orcid.org/0000-0002-9108-2990 Received: 27.05.2018 Accepted: 15.09.2018 


\section{Discussion}

The female urethra is $4-5 \mathrm{~cm}$ in length with a diameter of 5-6 $\mathrm{mm}$. After extending down the anterior wall of the vagina, its external orifice is located about $2.5 \mathrm{~cm}$ behind the clitoris. The urethra is present in both sexes and its main function is urine excretion (4). Disorders of female urethral meatus are quite varied; in addition, the vulva and vagina are also affected by their own diseases. Histologically, the parts closer to the bladder comprise transitional multilayered non-keratinized and occasionally glandular epithelium. Malignant masses of the urethra are very rare and include transitional cell carcinoma, squamous cell carcinoma, adenocarcinoma. And among the benign urethral masses are polyps, papillomas, hemangiomas, fibroma, leiomyomas, fibromyomas and adenomas (5). While it is a fact that leiomyomas are seen rarely, polyps and papillomas are the most frequently seen benign tumors.

Leiomyomas which origin from smooth muscles are benign mesenchymal tumors $(6,7,8)$. In women of birth age, they are the most frequently seen uterine masses. But, there have also been cases in which they have occurred in uncommon sites and displayed unusual growth patterns (9). The sites where extrauterine leiomyomas most frequently develop are vulva, ovaries, urethra, and bladder in the genitourinary tract or where less frequently develop is in the gastrointestinal system, but can occur in almost any tissue that contains smooth muscle. They mainly affect women between the ages of 30-50 years (7). There are debates about their degree of estrogen dependence, and diagnosis is made by histopathological examination of the relevant tissue (6). Urethral leiomyomas grow in a hormonedependent manner like uterine leiomyomas, and may also express estrogen and progesterone receptors (10). In women of reproductive age, they are inclined to enlarge during pregnancy and regress in the postpartum period (11). Whereas the anterior wall of the vagina is the site where paraurethral leiomyomas develop, the anterior wall of the proximal urethra is where urethral leiomyomas most frequently do (12).

Patients with urethral leiomyomas are usually asymptomatic, but sometimes an enlarged mass on the anterior vaginal wall obstructs the urethral meatus and causes obstructive symptoms. Common symptoms include dysuria, weak urine flow, mass, dyspareunia, post-voiding drip, frequent bowel movements, and irritative lower urinary system symptoms. Occasionally, patients present with recurrent urinary tract infections $(13,14)$. It should be kept in mind that acute urinary retention may develop in complete stenosis. Malignant degeneration and metastasis have not yet been reported (15). In case urethral strictures develop, urethral and paraurethral lesions need to be removed with caution in order to avoid postoperative incontinence much as vulvar lesions have not been linked with important postoperative complications so far. Leiomyoma should be included in diagnostic evaluations in patients with urethral stricture. It should be considered in the differential diagnosis of intraurethral neoplasms. As the surgical approach may differ accordingly it is also important to distinguish whether they are urethral or periurethral masses.

In majority of the cases in the literature, urethral leiomyomas have been defined as intraluminal masses which stem from the proximal urethra and extending outward from the meatus to the posterior (16). In our case, the leiomyoma was overwhelmingly a perineal mass which extended downward from the distal urethra, and resulted from the anterior urethral wall. A mass originating from the anterior wall of the vagina may cause dysuria by occluding the urethral meatus. In our case, the leiomyoma did not invade the urethra, but caused intense dysuria. These complaints disappeared after surgical treatment. The kidneys and bladder are the urinary system organs most commonly affected by leiomyomas, while the urethra is a rare location for such tumors $(14,15)$. The etiopathogenesis of leiomyoma is unknown; however it is thought to be hormonedependent to a certain degree. This theory is supported by evidence of tumor growth during pregnancy described by some authors and the presence of smooth muscle hyperplasia, which is stimulated by high estrogen levels. On the other hand, other authors reject the hormonal origin of urethral leiomyomas, citing the fact that some cases occur in postmenopausal women or that existing tumors are non-invasive (8). The patient in our case was a fertile woman, which may support the hormonal origin of smooth muscle hyperplasia in the development of leiomyomas.

In terms of macroscopic appearance, the tumor is usually a hard, round, smooth, pink lesion. Although its diameter ranges from $1 \mathrm{~cm}$ to $3-4 \mathrm{~cm}$, a case with a diameter of $40 \mathrm{~cm}$ has been reported (6). The clinical symptoms that occur during this

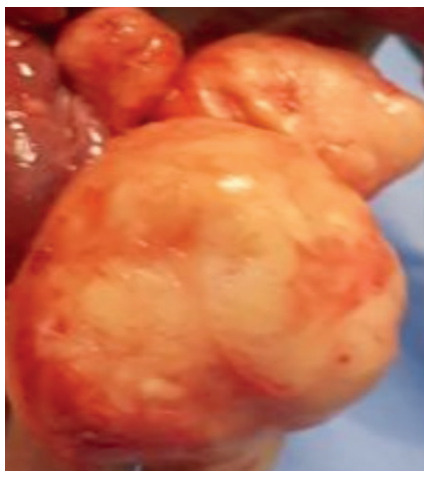

Figure 1. Macroscopic appearance following surgical removal

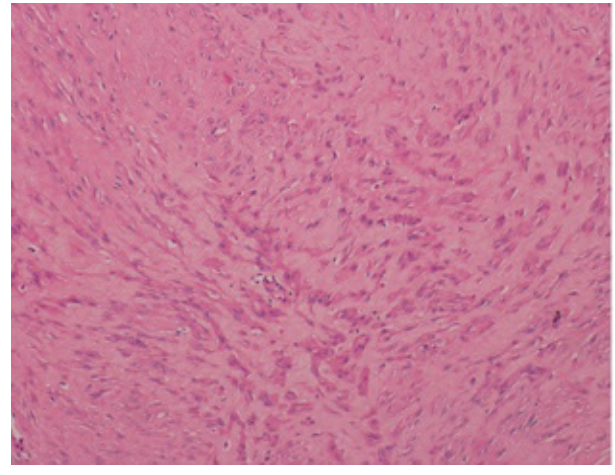

Figure 2. Microscopic appearance with hematoxylin and eosin staining

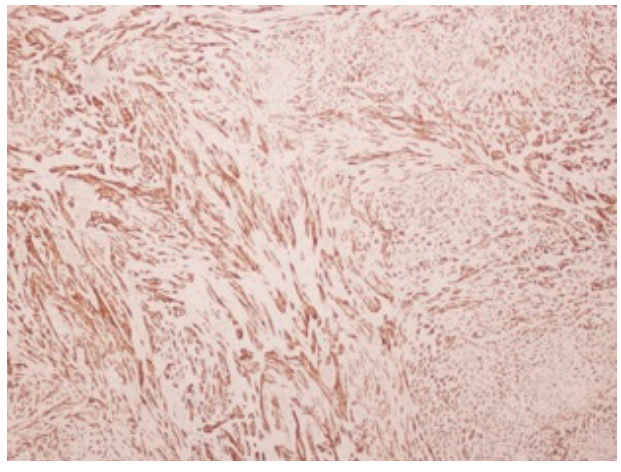

Figure 3. Microscopic appearance with desmin staining 
process depend on the site and size of the tumor. In the clinical diagnosis of this disease, a complete physical examination of the vulva and thorough history taking are quite important. While a preliminary diagnosis can be made visually by external USG and magnetic resonance imaging, conclusive diagnosis is reached after the histopathological confirmation (6). Leiomyomas have a characteristic myomic appearance in USG (16).

The treatment of urethral leiomyoma is always surgical, and the modality used depends on the level of urethral tumor. The prognosis for this type of tumor is excellent without malignant transformation $(3,8)$. The surgical approach depends on tumor size and location. Lower urinary tract symptoms regress following the complete removal of the urethral leiomyoma. The presence of a tumor on the anterior wall of the vagina damages the urethral mucosa, which results in dysuria and urethral hemorrhage. Following its surgical removal, the prognosis is excellent, generally with no malignant transformation. Our patient also had no complaints during the first postoperative year.

Physical examination of patients who present with dysuria, dyspareunia, and perineal mass should not be ignored, and benign masses such as leiomyoma should be kept in mind in the differential diagnosis. During surgical excision, care should be taken not to damage the urethra, and an indwelling urinary catheter should be left in place for an extended time to prevent complications such as stenosis or fistula.

\section{Ethics}

Informed Consent: It was obtained.

Peer-review: Internally peer-reviewed.

\section{Authorship Contributions}

Surgical and Medical Practices: M.A., M.G., M.S., Concept: M.A., S.Ü., Design: S.Ü., M.A., Data Collection or Processing: M.A., M.G., M.S., Analysis or Interpretation: M.A., M.G., M.S., Literature Search: M.A., M.G., M.S., S.Ü., Writing: M.A., M.G., M.S., S.Ü.

Conflict of Interest: No conflict of interest was declared by the authors.

Financial Disclosure: The authors declared that this study received no financial support.

\section{References}

1. Sohn GS, Cho S, Kim YM, et al. Current medical treatment of uterine fibroids. Working Group of Society of Uterine Leiomyoma. Obstet Gynecol Sci 2018;61:192-201.

2. Buttner C. Ein fall von Myom der Weiblichen Urethra [A case of myoma of the female urethra]. Z Geburshc Gynak. 1894;28:135-136.

3. Fridman D, Abeshouse M, Sankin A. Paraurethral Leiomyoma as an Incidental Finding in Patient with Fibroid Uterus. Case Rep Obstet Gynecol 2018;2018:7042960.

4. Lee MC, Lee SD, Kuo HT, Huang TW. Obstructive leiomyoma of the female urethra: report of a case. J Urol 1995;153:420-421.

5. Shield DE, Weiss RM. Leiomyoma of the female urethra. J Urol 1973;109:430-431.

6. Marchitelli C. Pseudotumores. Enfermedades de la Vulva y Vagina. Disponible en: http:www.enfermedaddelavulva.com.ar/Claudia. marchitelli@enfermedaddelavulva.com.ar. 2007.

7. Leung YL, Lee F, Tam PC. Leiomioma of the female urethra causing acute urinary retention and acute renal failure. J Urol 1997;158:1 911-912.

8. Vallmanya Llena FR, Rijo Mora E, Hernández Pozo H, et al. [Urethral leiomyoma]. Actas Urol Esp 2007;31:1196.

9. Fasih N, Prasad Shanbhogue AK, Macdonald DB, et al. Leiomyomas beyond the uterus: unusual locations, rare manifestations. Radiographics 2008;28:1931-1948.

10. Alvarado-Cabrero I, Candanedo-González F, Sosa-Romero A. Leiomyoma of the urethra in a Mexican woman: a rare neoplasm associated with the expression of estrogen receptors by immunohistochemistry. Arch Med Res 2001;32:88-90.

11. Fry $M$, Wheeler JS Jr, Mata JA, et al. Leiomyoma of the female urethra. J Urol 1988; 140:613-614.

12. Ozel B, Ballard C. Urethral and paraurethral leiomyomas in the female patient. Int Urogynecol J Pelvic Floor Dysfunct 2006;1 7:93-95.

13. Fedelini P, Chiancone F, Fedelini $M$, et al. A very large leiomyoma of the urethra: a case report. Urologia 2018;85:79-82.

14. Beng Kwang N, Naidu A, Yahaya A, Pei Shan L. Urethral Leiomyoma: A Rare Clinical Entity. Case Rep Surg 2016;2016:6037104.

15. Vilar G, Martin JP, Aguado M, et al. Leiomyoma of the female urethra: a case report and review of the literature. Actas Urol ESP 2010;34:396-397.

16. Lee MC, Lee SD, Kuo HT, Huang TW. Obstructive leiomyoma of the female urethra: report of a case. J Urol 1995;153:420-421. 